\title{
Renal Insufficiency and Short-Term Outcomes of Acute Pulmonary Embolism: A Systemic Review and Meta-Analysis
}

\author{
Dingyi Wang ${ }^{1,2}$ Guohui Fan ${ }^{1,2}$ Xin Liu ${ }^{3}$ Sinan Wu ${ }^{1,2}$ Zhenguo Zhai ${ }^{1,4}$
}

\footnotetext{
${ }^{1}$ Institute of Clinical Medical Sciences, China-Japan Friendship Hospital, Beijing, China

2 Institute of Respiratory Medicine, National Clinical Research Center for Respiratory Disease, Chinese Academy of Medical Sciences, Beijing, China

${ }^{3}$ Department of Nephrology, Beijing Hospital, National Center of Gerontology, Institute of Geriatric Medicine, Chinese Academy of Medical Sciences, Beijing, China

${ }^{4}$ Department of Pulmonary and Critical Care Medicine, Center of Respiratory Medicine, China-Japan Friendship Hospital, Beijing, China
}

Thromb Haemost 2020;120:1025-1034.

\begin{abstract}
Address for correspondence Sinan Wu, MD, Institute of Clinical Medical Sciences, China-Japan Friendship Hospital, Beijing, China; Institute of Respiratory Medicine, National Clinical Research Center for Respiratory Disease, Chinese Academy of Medical Sciences, Beijing, China (e-mail: tks0423@hotmail.com).
\end{abstract}

Zhenguo Zhai, MD, PhD, Department of Pulmonary and Critical Care Medicine, Center of Respiratory Medicine, China-Japan Friendship Hospital, Peking University Health Science Center, Beijing, China (e-mail: zhaizhenguo2011@126.com).

\begin{abstract}
Keywords

- pulmonary embolism

- venous thromboembolism

- renal insufficiency

- acute kidney injury

- mortality
\end{abstract}

Background This article evaluates the association between renal insufficiency and short-term outcomes among patients with acute pulmonary embolism.

Methods The literature search was completed on December 31, 2019 and data were contracted from 13 cohort studies. Diagnosis of renal insufficiency was based on estimated glomerular filtration rate (eGFR), serum creatinine level, or self-report. The primary outcome was all-cause mortality of 30 days or during hospitalization. The pooled risk ratios (RRs), pooled mortality rates, and between-study heterogeneity were estimated by random-effect models. All the statistical analyses were performed using STATA/SE software.

Results We included 13 studies $(N=35,662)$ in the meta-analysis, including two focused on acute kidney injury (AKI). Early all-cause mortality in patients with versus without renal insufficiency were 15\% (95\% confidence interval [CI] 9-22\%) and 5\% (95\% Cl 3-8\%), respectively (RR $1.76,95 \% \mathrm{Cl} 1.61-1.92)$. For patients with eGFR $<30$ $\mathrm{mL} / \mathrm{min} \cdot 1.73 \mathrm{~m}^{-2}$, rates were $30 \%(95 \% \mathrm{Cl} 11-75 \%)$ versus $10 \%(95 \% \mathrm{Cl} 5-14 \%)$ (RR 3.32, $95 \% \mathrm{Cl} 1.53-6.70)$. For patients with AKI during hospitalization, rates were $32 \%$ (95\% Cl 11-75\%) versus 13\% (95\% Cl 4-29\%) (RR 2.69, 95\% Cl 1.24-5.84). Pulmonary embolism (PE)-related death and fatal bleeding were significantly higher in patients with renal insufficiency.

Conclusion Renal insufficiency, especially AKI and severe renal insufficiency, was associated with early mortality in acute PE patients. Our results may escalate vigilance in risk stratification and management of PE patients with renal insufficiency in clinical practice. received

March 12, 2020

accepted after revision

April 16, 2020 (c) 2020 Georg Thieme Verlag KG Stuttgart . New York
DOI https://doi.org/ 10.1055/s-0040-1712459. ISSN 0340-6245. 


\section{Introduction}

Acute pulmonary embolism (PE) contributes to the global burden in terms of morbidity mortality, and financial impact on health care systems. ${ }^{1}$ A reliable risk classification and prediction model of PE is important for identifying patients with higher risk of short- or long-term adverse outcomes, thus affects clinical decision-making. Pulmonary Embolism Severity Index (PESI) or simplified PESI (SPESI) ${ }^{1}$ are recently reliable in assessments of 30-day outcome of patients with acute PE and have been wildly validated. ${ }^{2-4}$ sPESI score focused on 6 equally weighed variables-age $>80$ years, cancer, chronic heart failure or chronic pulmonary disease, systolic blood pressure $<100 \mathrm{~mm} \mathrm{Hg}$, and arterial oxyhemoglobin saturation $<90 \% .{ }^{5}$ Whereas several studies suggested that the clinical severity scores alone might be insufficient to accurately stratify the full spectrum of disease, biomarkers are beneficial for further identification of patients in different risk of outcomes. 6,7

Renal dysfunction is one of the generally accepted indications of an increased mortality in various cardiovascular diseases. Several large registries have demonstrated the impact of renal function in both short- and long-term prognosis of acute $\mathrm{PE}$. In the International Cooperative Pulmonary Embolism Registry study, renal dysfunction (defined as creatinine level $>2.0 \mathrm{mg} / \mathrm{dL}$ ) was an independent predictor of mortality (hazard ratio [HR] 2.0; 95\% confidence interval [CI] 1.4-3.0). ${ }^{8}$ In Registro Informatizado de Enfermedad TromboEmbólica (RIETE) study, creatinine clearance $(\mathrm{CrCl})<30 \mathrm{~mL} / \mathrm{min}$ was independently associated with an increased risk for fatal $\mathrm{PE}$ (odds ratio [OR] 5.2; 95\% CI 3.4-7.8) and fatal bleeding (OR 5.0; 95\% CI 2.0-12) within 15 days of diagnosis. ${ }^{9}$

Due to the different expression of renal function (e.g., creatinine level, $\mathrm{CrCl}$, or estimated glomerular filtration rate [eGFR] calculated by different formula) in separate studies, the association between renal insufficiency and the prognosis of acute PE is inconsistent. Therefore, we conducted the present meta-analysis to determine the prognostic relevance of renal insufficiency in patients with acute PE.

\section{Methods}

\section{Literature Search and Study Selection}

The literatures published in Pubmed, Web of Science, or EMBASE in the past 12 years from January 1, 2008, to December 31, 2019, was searched. The search terms included: kidney disease, kidney failure, kidney insufficiency, kidney function, kidney dysfunction, renal disease, renal failure, renal insufficiency, renal dysfunction, pulmonary embolism, pulmonary thromboembolism, thromboembolism, and venous thromboembolism (VTE). If appropriate, medical leading terms were used, such as MeSH and Emtree words.

We performed an additional manual search of potentially eligible studies within references of the included studies, international guidelines, relevant (systematic) reviews, and derivation/validation studies of prognostic scores.
Search results were screened independently by two reviewers for the relevance of titles/abstracts and full-texts of the studies fulfilling the inclusion criteria. Potential disagreements were solved by a third reviewer.

The present work was performed in accordance with the Preferred Reporting Items for Systematic Reviews and MetaAnalyses guideline. ${ }^{10}$

\section{Inclusion Criteria}

Studies were considered eligible if they fulfilled all the following criteria: (1) Patients should be included through objectively confirmed acute PE. (2) Data on renal function status at baseline or acute kidney injury (AKI) are available. (3) At least one of the outcomes of interest was assessed during short-term (in-hospital or less than 30 days) follow-up. (4) Publications must be in English language.

\section{Renal Function Measurement and Definition}

Renal function was estimated by the either of the following definitions:

eGFR by Modification of Diet in Renal Disease (MDRD):

$\mathrm{GFR}=186 \times \mathrm{Scr}^{-1.154} \times$ age $^{-0.203} \times 1.212($ if black $) \times 0.742$ (if female)

or Chronic Kidney Disease Epidemiology Collaboration (CKD-EPI) equation:

$\mathrm{GFR}=141 \times \min (\mathrm{Scr} / \mathrm{K}, 1)^{\alpha} \times \max (\mathrm{Scr} / \mathrm{K}, 1)^{-1.209}$ $\times 0.993^{\text {age }} \times 1.018$ (if female) $\times 1.159$ (if black)

The classification of renal dysfunction was based on the cutoffs reported in the selected studies.

AKI was determined according to the "Kidney Disease: Improving Global Outcomes" definition. ${ }^{11}$

Renal insufficiency was defined as self-reported "renal dysfunction," or creatinine elevation (according to particular studies), or eGFR $<60 \mathrm{~mL} / \mathrm{min} \cdot 1.73 \mathrm{~m}^{-2}$. Severe renal insufficiency was defined as eGFR $<30$ or $35 \mathrm{~mL} /$ $\min \cdot 1.73 \mathrm{~m}^{-2}$.

\section{Data Extraction and Quality Assessment}

Two researchers (D.W. and X.L.) independently extracted the following information from each study: lead author, publication year, country of origin, participant characteristics, measurement of renal function, classification of renal function, outcomes, and follow-up duration. The quality of studies was assessed in accordance with the Newcastle-Ottawa Scale for cohort studies. ${ }^{12}$

\section{Outcomes}

We defined short-term all-cause mortality (death during the hospital stay or within the first 30 days following diagnosis of $\mathrm{PE})$ as the primary outcome of our meta-analysis. The secondary outcomes were PE-related death, bleeding events (including major bleeding and fatal bleeding ${ }^{13}$ ), and adverse 
outcomes (defined as death, cardiopulmonary resuscitation or cardiogenic shock, use of vasopressors, thrombolysis, mechanical ventilation, etc.).

\section{Statistical Analysis}

We estimated pooled relative risk (RR) and 95\% CI and weighed rates between groups of patients with and without renal dysfunction using a random-effect model. We assessed statistical heterogeneity of exposure effects by calculating the inconsistency index $I^{2}$ statistic (ranging from 0 to $100 \%$ ) on the basis of the Cochrane $Q$ test, which summarizes the amount of variance among studies beyond chance. Heterogeneity was defined as low $\left(I^{2}<25 \%\right)$, moderate $\left(I^{2}=25-\right.$ $75 \%)$, or high $\left(I^{2}>75 \%\right)$. The presence of publication bias was evaluated by visually inspecting funnel plots. Meanwhile, the Egger's test, a weighted regression test helping justify the asymmetry of funnel plots, was performed to assess the statistical evidence of publication bias, with a significance level defined as $p<0.1$.

We performed predefined subgroup analyses investigating the prognostic value of different cutoffs, stratifications, and study designs. STATA software (StataCorp, Texas, United States, version 14.0 for Windows) was served for data analysis.

\section{Results}

The literature search identified 1,849 records in Pubmed, 1,876 in Web of Science, and 5,641 in EMBASE, leaving 2,732 records after duplicate removing. - Fig. 1 shows the selection flowchart of the meta-analysis. Eventually, we include 13 studies in the meta-analysis with a total of 35,662 patients diagnosed with acute PE (-Table 1), 2 of them were conference abstracts and the others were published articles. Of these studies, baseline renal function stratification was available in 11 studies ( 7 measured by eGFR, ${ }^{14-21} 2$ by serum creatinine level, ${ }^{22,23}$ and 2 by self-report ${ }^{24,25}$ ), and data on AKI occurrence after hospitalization was available in 2 studies. $^{26,27}$

\section{Short-Term Mortality}

The analysis of renal insufficiency was reported from 7 studies in 1,761 of 5,364 patients (32.8\%). Short-term allcause mortality among those patients were $15 \%$ (95\% CI 9-22\%) and 5\% (95\% CI 3-8\%), respectively (RR 1.76, 95\% CI $\left.1.61-1.92 ; I^{2} 0 \%\right)$. Subgroup analysis of short-term all-cause mortality showed patients with creatinine elevation had a pooled RR of 2.18 (95\% CI 1.41-3.36), patients with eGFR $<60 \mathrm{~mL} / \mathrm{min} \cdot 1.73 \mathrm{~m}^{-2}$ had a pooled RR of $1.72(95 \%$ CI 1.56-1.89) (- Table 2, - Fig. 2).

Severe renal insufficiency was reported in 2 studies with 206 out of 3,508 patients (5.9\%). Short-term all-cause mortality among those patients were $30 \%$ (95\% CI 2-59\%) and 10\% (95\% CI 5-14\%), respectively (RR 3.32, 95\% CI 1.53-6.70; $I^{2}$ 80.7\%) (-Fig. 3).

AKI after hospitalization was reported from 2 studies in 6,594 out of 28,719 patients (23.0\%). Short-term all-cause mortality among those patients were $32 \%$ (95\% CI 11-75\%)

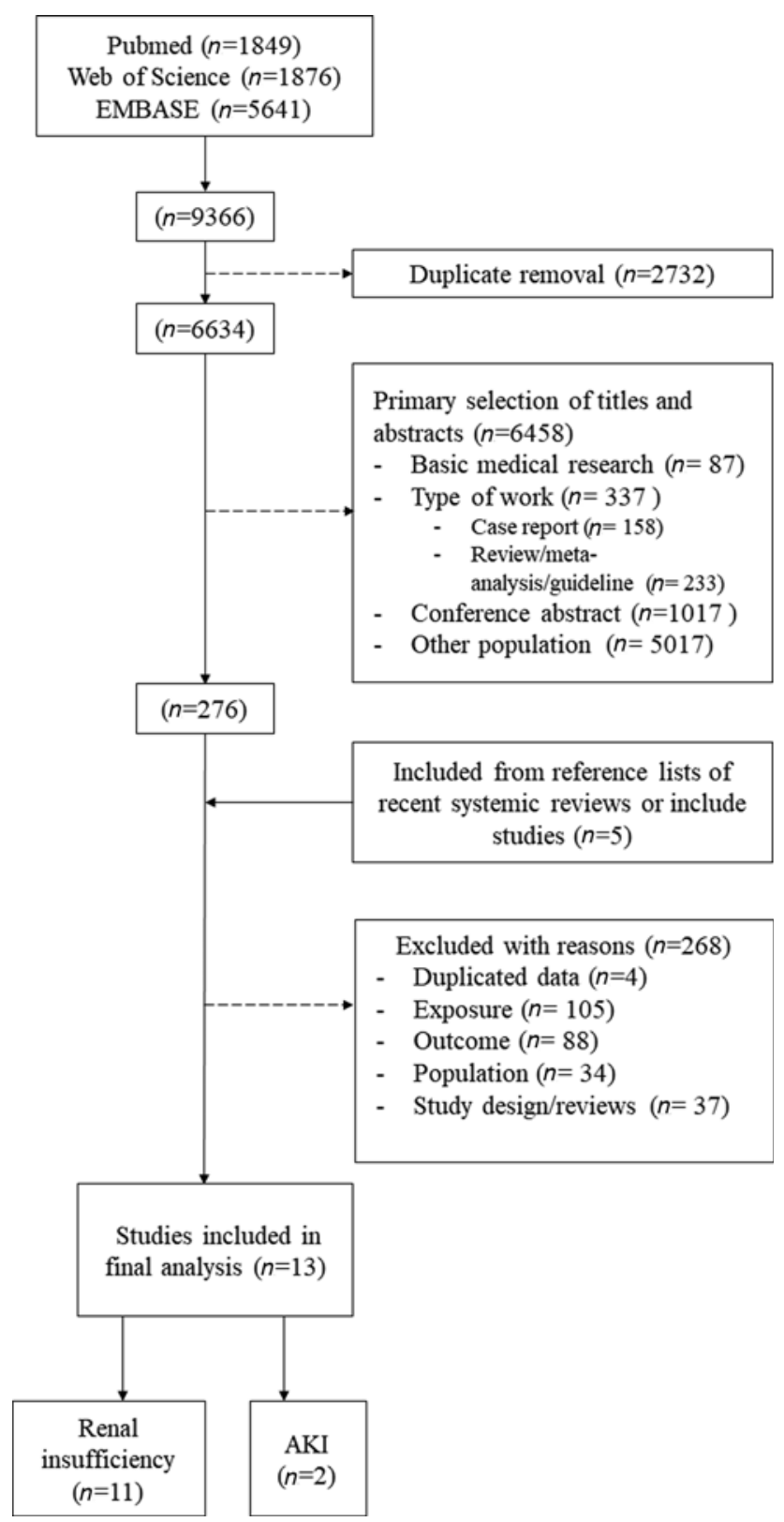

Fig. 1 Flowchart of study selection. AKI, acute kidney injury.

and 13\% (95\% CI 4-29\%), respectively (RR 2.69, 95\% CI 1.24-5.84; $I^{2}$ 98.2\%) (-Fig. 4A).

\section{Secondary Outcomes}

PE-related mortality was higher in patients with renal insufficiency (12\% vs. 4\%, RR 1.72, 95\% CI 1.55-1.91; $I^{2} 0 \%$ ) (-Fig. 5A).

Fatal bleeding was available as a secondary outcome in two studies. Patients with renal insufficiency had higher risk of fatal bleeding (RR 1.43, 95\% CI 1.22-1.67; $I^{2}$ 0\%) (- Fig. 5B).

Adverse outcomes were available as a combined endpoint in three studies. Patients with renal insufficiency had higher incidence but the risk ratio was not statistically significant (22\% vs. $12 \%$, RR 2.64, 95\% CI 0.80-8.58).

Major bleeding was described in the two studies focused on AKI. The risk of major bleeding among AKI patients was not significantly higher than those without AKI (-Fig. 3B). 


\begin{tabular}{|c|c|c|c|c|c|c|c|c|c|c|c|c|c|c|c|}
\hline 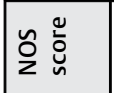 & 0 & 0 & $r$ & 0 & 1 & $\wedge$ & $\Sigma$ & $\frac{\pi}{z}$ & $\wedge$ & r & $\wedge$ & r & $\Sigma$ & r & 0 \\
\hline 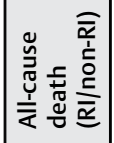 & $\underset{\sim}{\stackrel{N}{J}}$ & $\Sigma$ & $\frac{\sqrt{2}}{\frac{d}{2}}$ & $\stackrel{ \pm}{=}$ & 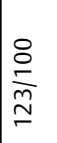 & $\stackrel{\frac{9}{0}}{0}$ & $\frac{\sqrt{a}}{\frac{\pi}{2}}$ & 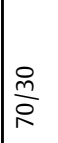 & $\Sigma$ & $\Sigma$ & $\Sigma$ & 量 & $\underset{\stackrel{N}{\infty}}{\stackrel{N}{\infty}}$ & $\begin{array}{l}\tilde{0} \\
0 \\
\frac{0}{f} \\
\tilde{N}\end{array}$ & 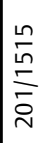 \\
\hline $\begin{array}{l}\widetilde{a} \\
\text { ज्ञ } \\
\stackrel{0}{\square}\end{array}$ & 文 & ఠ & $\Sigma$ & 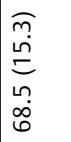 & 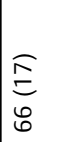 & 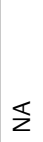 & 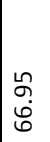 & $\Sigma$ & 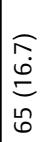 & $\stackrel{\infty}{\bullet}$ & 8 & 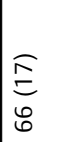 & & & 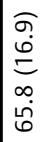 \\
\hline 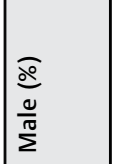 & 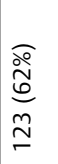 & 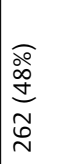 & 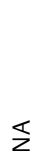 & 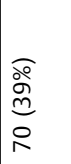 & 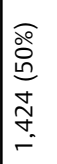 & 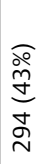 & 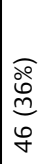 & $\Sigma$ & $\begin{array}{l}\text { } \\
\stackrel{\circ}{0} \\
\text { ñ } \\
\text { in }\end{array}$ & 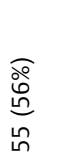 & 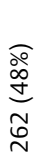 & 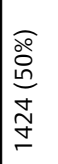 & & 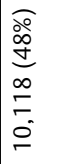 & 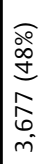 \\
\hline 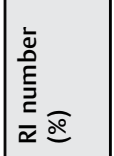 & 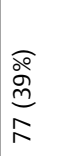 & 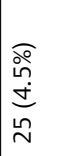 & 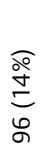 & $\begin{array}{l}\text { ò } \\
\text { ป̀ } \\
\text { o }\end{array}$ & 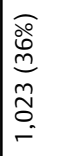 & 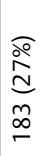 & 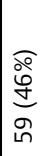 & 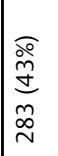 & $\begin{array}{l}\stackrel{\circ}{\stackrel{\circ}{9}} \\
\underline{\sigma}\end{array}$ & $\begin{array}{l}\widehat{\circ} \\
\infty \\
\stackrel{\infty}{0} \\
\infty \\
\infty \\
m\end{array}$ & 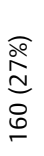 & 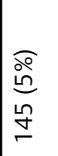 & $\frac{\sqrt{9}}{6}$ & 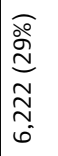 & 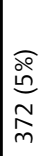 \\
\hline 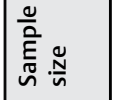 & 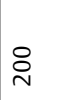 & 守 & ठ̊. & 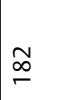 & $\begin{array}{l}\stackrel{n}{0} \\
\infty \\
\sim \\
\sim\end{array}$ & $\stackrel{\infty}{6}$ & $\stackrel{\mathscr{I}}{\stackrel{1}{2}}$ & : & $\underset{\text { Nి }}{\text { Dे }}$ & g & 尺̊ & $\mid \begin{array}{l}n \\
\infty \\
\infty \\
i\end{array}$ & 莕 & $\frac{\bar{m}}{\bar{i}}$ & 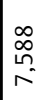 \\
\hline 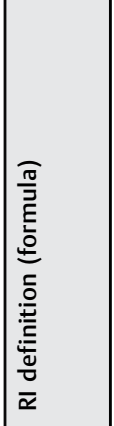 & 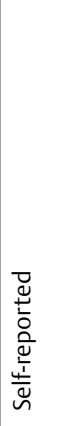 & 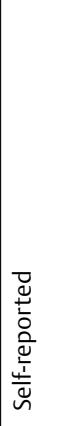 & 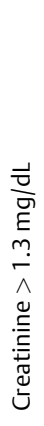 & 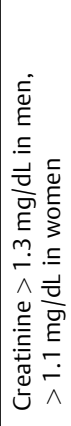 & 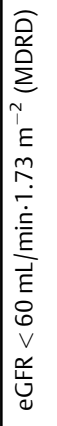 & 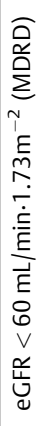 & 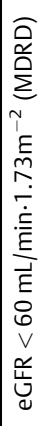 & 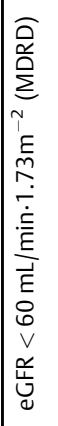 & 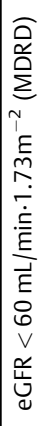 & 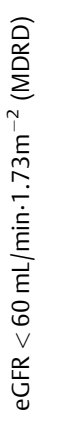 & 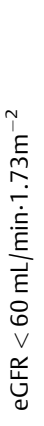 & 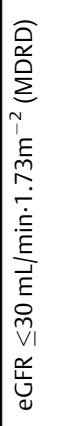 & 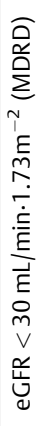 & 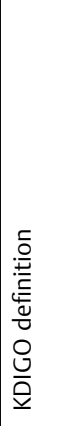 & $\underline{z}$ \\
\hline
\end{tabular}

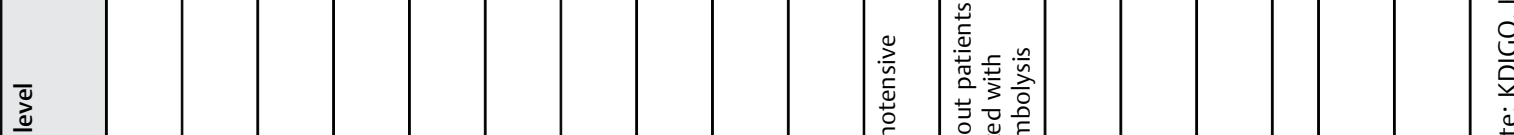

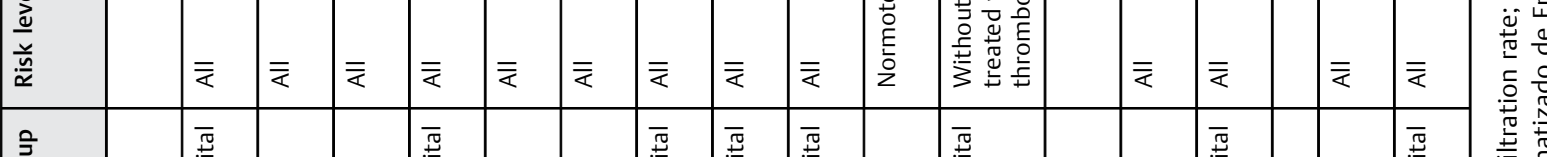

\begin{tabular}{|c|c|c|c|c|c|c|c|c|c|c|c|c|c|c|c|}
\hline 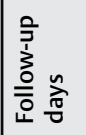 & 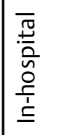 & 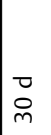 & : & 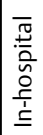 & : & $\begin{array}{l}\tilde{D} \\
\dot{m}\end{array}$ & 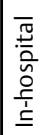 & 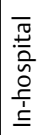 & 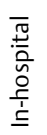 & ¿্口 & 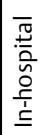 & 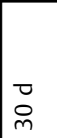 & 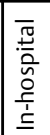 & 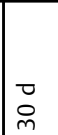 & 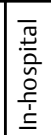 \\
\hline
\end{tabular}

\begin{tabular}{|c|c|c|c|c|c|c|c|c|c|c|c|c|c|c|c|c|c|c|}
\hline 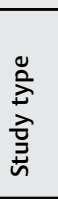 & & 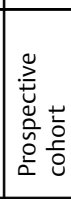 & 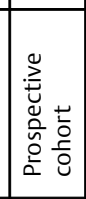 & 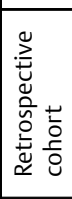 & 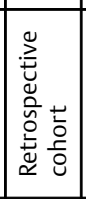 & 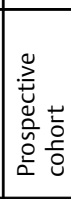 & 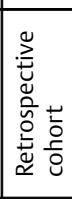 & 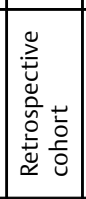 & 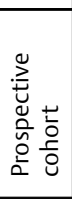 & 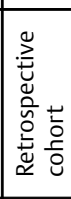 & 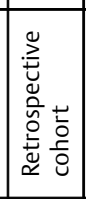 & 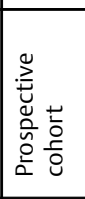 & & 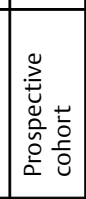 & 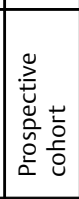 & & 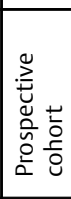 & 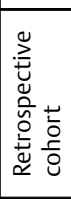 \\
\hline 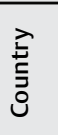 & & 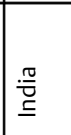 & 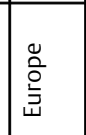 & 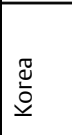 & 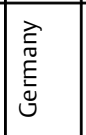 & 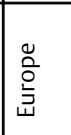 & 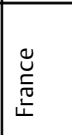 & 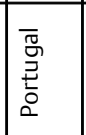 & 茜 & 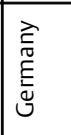 & \begin{tabular}{|l} 
⿹े \\
咅
\end{tabular} & 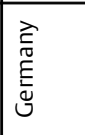 & & 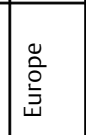 & 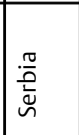 & & 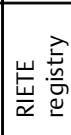 & 蛋 \\
\hline 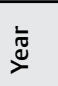 & & 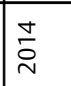 & 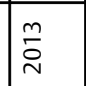 & 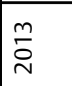 & 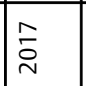 & $\frac{\stackrel{9}{\nu}}{\grave{N}}$ & $\underset{\sim}{\dot{D}}$ & $\stackrel{\stackrel{n}{2}}{\stackrel{N}{N}}$ & $\stackrel{g}{\stackrel{N}{N}}$ & $\underset{\sim}{\sim}$ & 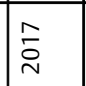 & 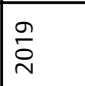 & & 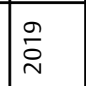 & 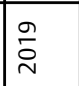 & & 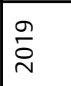 & 产 \\
\hline & 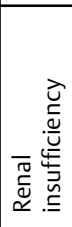 & 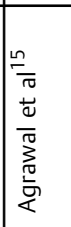 & 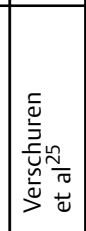 & $\begin{array}{l}\frac{\pi}{\sqrt{0}} \\
\tilde{J} \\
0 \\
0\end{array}$ & 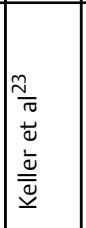 & 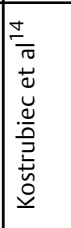 & 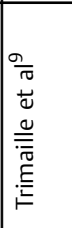 & 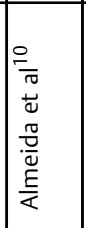 & 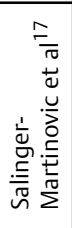 & 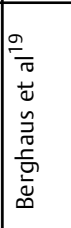 & 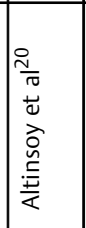 & 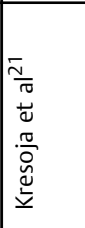 & 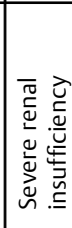 & 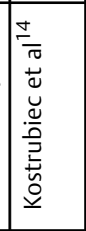 & 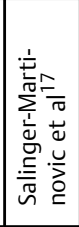 & \begin{tabular}{|l} 
\\
$\bar{z}$
\end{tabular} & 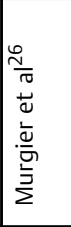 & 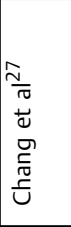 \\
\hline & & - & $\sim$ & $m$ & 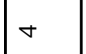 & in & 0 & $\wedge$ & $\infty$ & $a$ & $\circ$ & $=$ & & 几 & $\infty$ & & $\simeq$ & $\stackrel{m}{m}$ \\
\hline
\end{tabular}


Table 2 Pooled rates and RRs of short-term outcomes of patients with/without renal insufficiency

\begin{tabular}{|c|c|c|c|}
\hline & $\begin{array}{l}\text { With renal insufficiency/ } \\
\text { rate }(95 \% \mathrm{Cl})\end{array}$ & $\begin{array}{l}\text { Without renal insufficiency/ } \\
\text { rate }(95 \% \mathrm{Cl})\end{array}$ & RR (95\% Cl) \\
\hline \multicolumn{4}{|l|}{ All-cause mortality } \\
\hline Self-reported renal disease & $31 \%(21 \%, 42 \%)$ & $10 \%(5 \%, 15 \%)$ & $2.06(1.50,2.84)$ \\
\hline Creatinine elevation & $10 \%(5 \%, 25 \%)$ & $5 \%(1 \%, 9 \%)$ & $2.18(1.41,3.36)$ \\
\hline $\mathrm{eGFR}<60 \mathrm{~mL} / \mathrm{min} \cdot 1.73 \mathrm{~m}^{-2}$ & $15 \%(8 \%, 22 \%)$ & $5 \%(2 \%, 8 \%)$ & $1.72(1.56,1.89)$ \\
\hline Severe renal insufficiency & $30 \%(2 \%, 59 \%)$ & $10 \%(5 \%, 14 \%)$ & $3.32(1.53,6.70)$ \\
\hline AKI & $32 \%(11 \%, 75 \%)$ & $13 \%(4 \%, 29 \%)$ & $2.69(1.24,5.84)$ \\
\hline \multicolumn{4}{|l|}{ PE-related death } \\
\hline $\mathrm{eGFR}<60 \mathrm{ml} / \mathrm{min} \cdot 1.73 \mathrm{~m}^{-2}$ & $12 \%(6 \%, 18 \%)$ & $4 \%(2 \%, 5 \%)$ & $1.72(1.55,1.91)$ \\
\hline \multicolumn{4}{|l|}{ Fatal bleeding } \\
\hline Self-reported renal disease & NA & NA & NA \\
\hline Creatinine elevation & NA & NA & NA \\
\hline eGFR $<60 \mathrm{~mL} / \mathrm{min} \cdot 1.73 \mathrm{~m}^{-2}$ & $7 \%(0,21 \%)$ & $0(0,1 \%)$ & $1.43(1.22,1.67)$ \\
\hline AKI & $5 \%(1 \%, 9 \%)$ & $4 \%(0,10 \%)$ & $1.30(0.79,2.14)$ \\
\hline \multicolumn{4}{|l|}{ Adverse outcomes } \\
\hline $\mathrm{eGFR}<60 \mathrm{ml} / \mathrm{min} \cdot 1.73 \mathrm{~m}^{-2}$ & $22 \%(12 \%, 32 \%)$ & $12 \%(0 \%, 26 \%)$ & $2.64(0.80,8.68)$ \\
\hline
\end{tabular}

Abbreviations: AKI, acute kidney injury; eGFR, estimated glomerular filtration rate; NA, not available; RR, risk ratio; $95 \% \mathrm{Cl}$, $95 \%$ confidence interval.

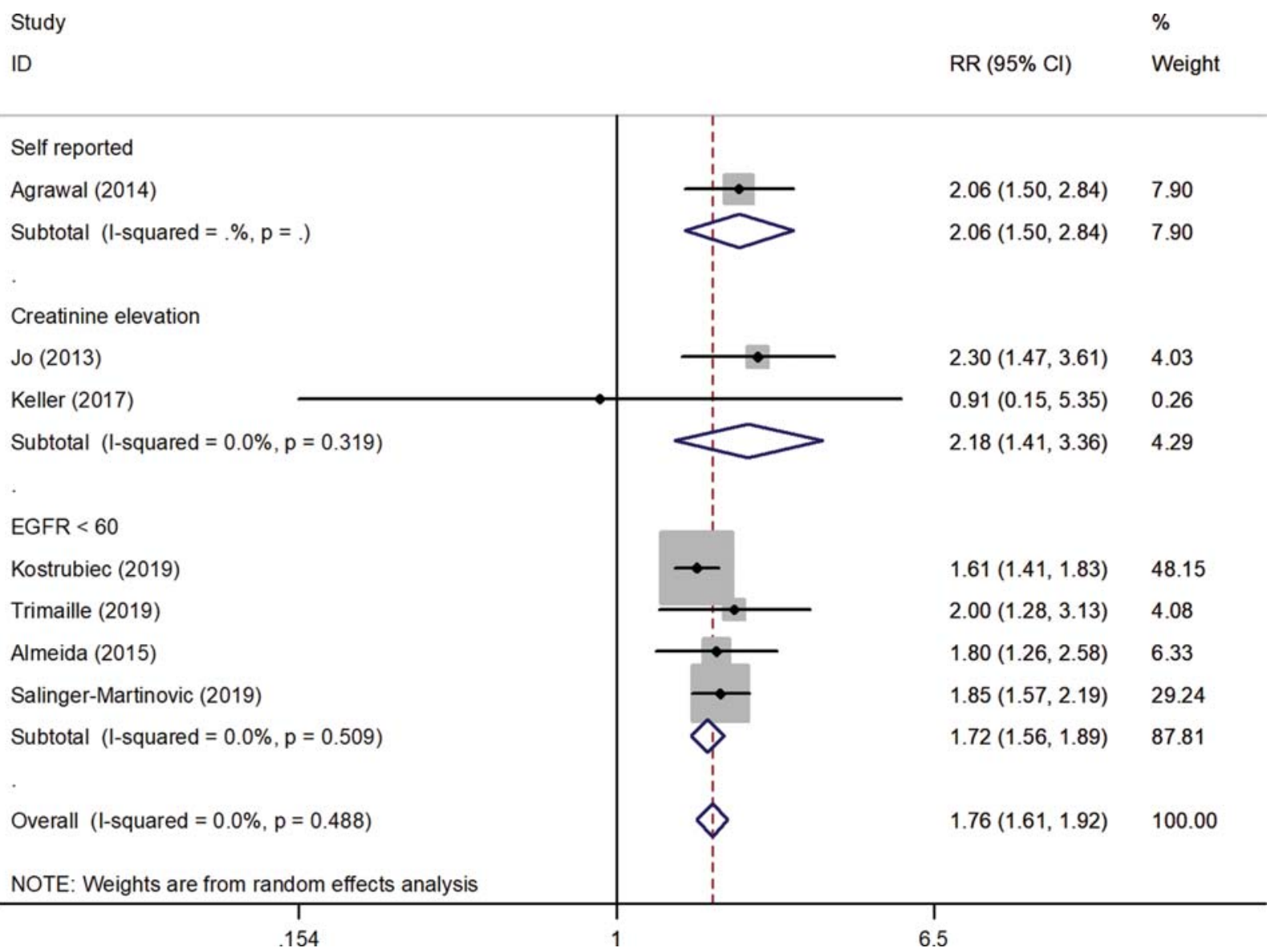

Fig. 2 Overall forest plot of renal insufficiency and all-cause death in pulmonary embolism (PE) patients. 95\% Cl, 95\% confidence interval; eGFR, estimated glomerular filtration rate; RR, risk ratio. 


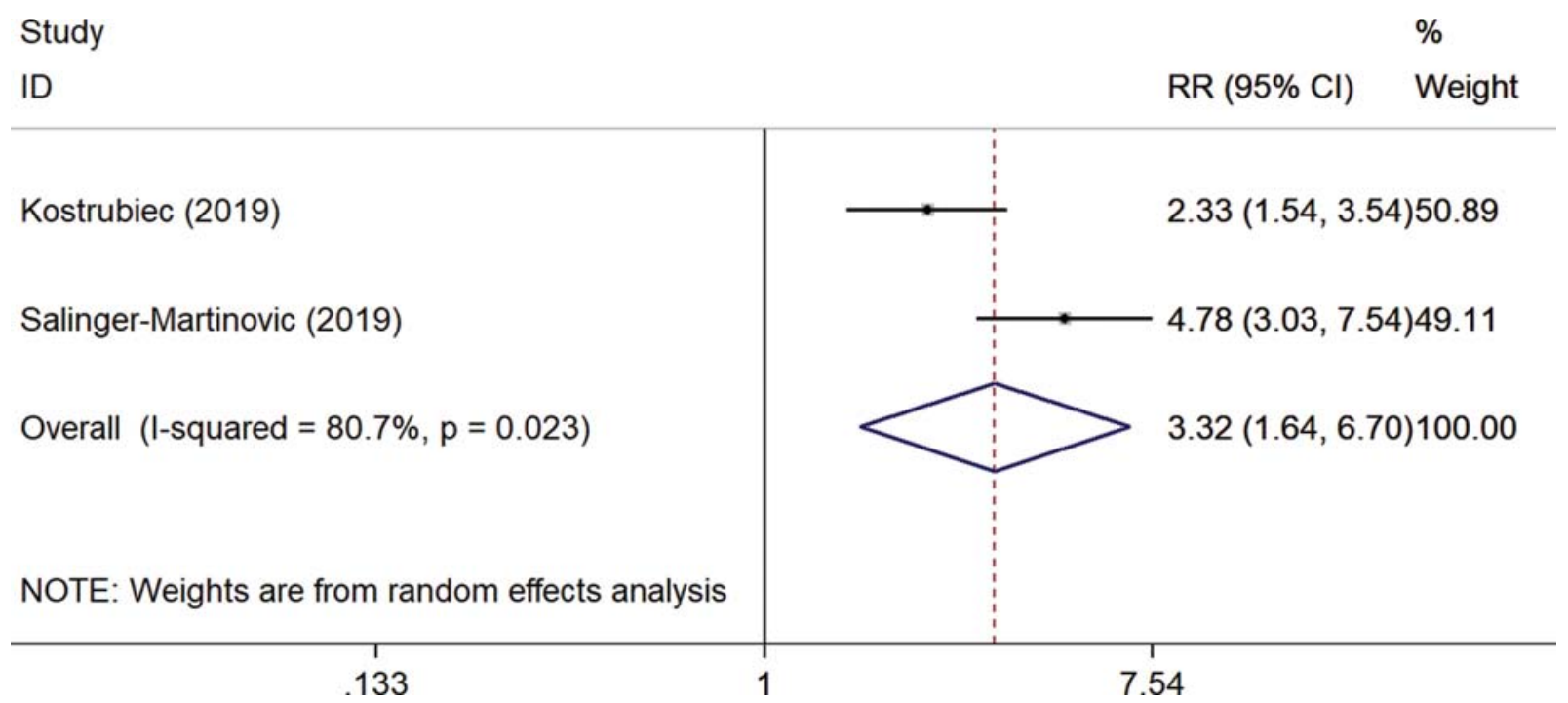

Fig. 3 Forest plot of all-cause death in pulmonary embolism (PE) patients with severe renal insufficiency.

\section{Outcomes of Normotensive Patients}

Three studies focused on normotensive or patients without thrombolysis. The outcomes are listed in $\boldsymbol{- T a b l e ~} \mathbf{3}$. All-cause death, major bleeding, and adverse outcomes were higher in patients with renal insufficiency. Pooled RRs were not able to be calculated as the outcomes differed in each study.

\section{Publication Bias}

According to the funnel plot (-Fig. 6) and the asymmetry statistic of Egger's regression, the probability of publication bias was low (Egger's test $p=0.583$ ).

\section{Discussion}

The results of the present meta-analysis indicate that renal insufficiency was a common comorbidity (nearly one-third) among acute PE patients. Renal insufficiency and AKI may have a significant impact on the early negative prognosis of acute PE patients. PE patients with renal insufficiency also had higher risk in PE-related death and fatal bleeding. Patients with severe renal insufficiency had significantly high risk (over threefold) for short-term all-cause mortality. Our results may have implications for the acute-phase management of PE patients who appear to have CKD and also calls for more attention to the dynamic changes of kidney function during management, in addition to the guideline-suggested risk stratification.

The prevalence of renal insufficiency, severe renal insufficiency, and AKI in different studies were around 14 to $46 \%, 5$ to $9 \%$, and 5 to $29 \%$, respectively, indicating a high proportion of renal disease among PE patients. Several studies investigated an increasing risk for CKD patients to develop VTE. ${ }^{28,29}$ CKD patients are at risk of clot formation and risk of thrombosis. The mechanism includes increased level of procoagulant factors, decreased endogenous anticoagulants, and fibrinolytic activity. ${ }^{30}$ Impaired kidney function may reflect not only chronic renal disease but also deterioration secondary to hemodynamic disturbances. Renal function is not only closely associ- ated with preexisting renal pathology but also with hemodynamic alterations. However, it is difficult to distinguish between preexisting renal insufficiency and secondary kidney injury. ${ }^{31}$ Study from RIETE revealed that AKI was found almost in one-third of patients with PE and being more frequent and severe in patients with high-risk PE. ${ }^{26}$ Right ventricular dysfunction in PE patients causes central venous pressure increase, results in venous congestion, and leads to renal congestion then injury. ${ }^{32}$

Renal insufficiency has been reported to be a predictor of both early and long-term increased mortality in patients with VTE. Studies also demonstrated a long-term impact of renal failure in VTE patients. A study from RIETE showed that $\mathrm{CrCl}<50 \mathrm{~mL} / \mathrm{min}$ was an independent risk factor for 3month all-cause death in VTE patients (adjusted HR $1.83)^{33}$ Two of the studies included in our meta-analysis showed that patients with renal insufficiency had a HR of 2.31 (95\% CI 2.13-2.48) for 6-month all-cause mortality in PE patients. ${ }^{14}$ Other studies that focus on VTE patients demonstrated similar results. In GARFILED-VTE study, over 12month follow- up, patients with moderate- to-severe CKD were at an increased risk of all-cause mortality, major bleeding, and recurrent VTE. ${ }^{34}$

The balance between bleeding and thrombosis in CKD patients receiving anticoagulant drugs is particularly complicated. Drug dosage should be adjusted according to patients' renal function. It has been reported that longterm risk of mortality and bleeding increased in CKD patients on warfarin therapy. ${ }^{35}$ Ageno et al found that among 6,122 VTE patients, $38.1 \%$ were with moderate renal impairment and those patients were prescribed more nonvitamin $\mathrm{K}$ antagonist oral anticoagulants than those with normal renal function. ${ }^{36} \mathrm{CKD}$ has been considered as exclusion criteria in most of the randomized clinical trials. Thus, the high proportion of PE/VTE patients with CKD calls for more attention in routine clinical practice.

Our study indicated that renal function was associated with short-term PE outcome despite its different 


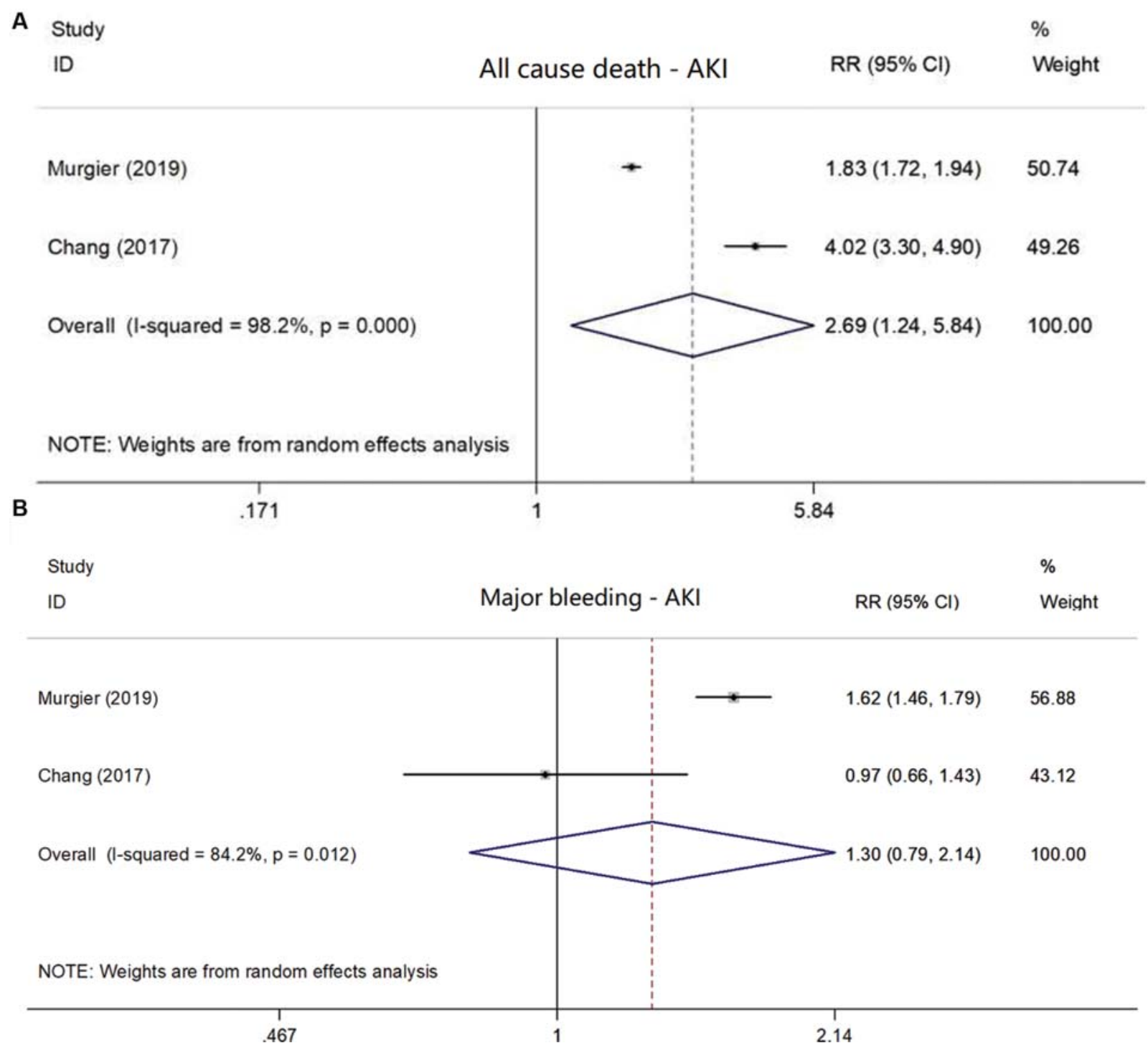

Fig. 4 Forest plots of all-cause death (A) and major bleeding (B) in pulmonary embolism (PE) patients with AKI. AKI, acute kidney injury; 95\% CI, $95 \%$ confidence interval; $R R$, risk ratio.

stratifications of involved studies. GFR is routinely used as an indicator of renal function. The most popular method for GFR estimation is the simplified MDRD equation, which takes into account serum creatinine levels, age, race, and gender. ${ }^{37}$ However, the CKD-EPI equation has been developed and reported to be more accurate than the MDRD formula. ${ }^{38}$ The studies recruited in our analysis used MDRD or $\mathrm{CrCl}$ to estimate the renal function status; even one study defined chronic renal diseases according to patients' self-report. All the three measurements-defined renal insufficiency showed significant influence to short-term adverse outcome in PE patients. Altinsoy et al suggested that the CKD-EPI formula had higher value in predicting adverse outcome in PE patients than MDRD formula, by comparing the two equations among a same cluster of patients. ${ }^{20}$ Some studies used serum creatinine level or the International Classification of Disease, 9th Revision, Clinical Modification to estimate renal function or define CKD and demonstrated no relationship between renal insufficiency and PE outcome, ${ }^{2,39}$ and some results were paradoxical. ${ }^{29}$ Thus, unified and accurate method to assess renal function in PE patients should be suggested.

To our knowledge, this is the first meta-analysis that focuses on renal function and short-term outcomes of acute PE. The recruited studies covered Europe, North America, and Asia. Our results call for attention to renal function, as well as its dynamic change during hospitalization of acute PE patients. Adding renal function to existing clinical severity score for acute PE is suggested. Meanwhile, the reason of the increased risk of death and bleeding should be further explained by investigating the correlation between treatment strategy and outcomes.

\section{Limitations}

Our meta-analysis has several limitations that need to be discussed. First, only 10 articles were included in the final analysis. Although there have been several ongoing large 
A

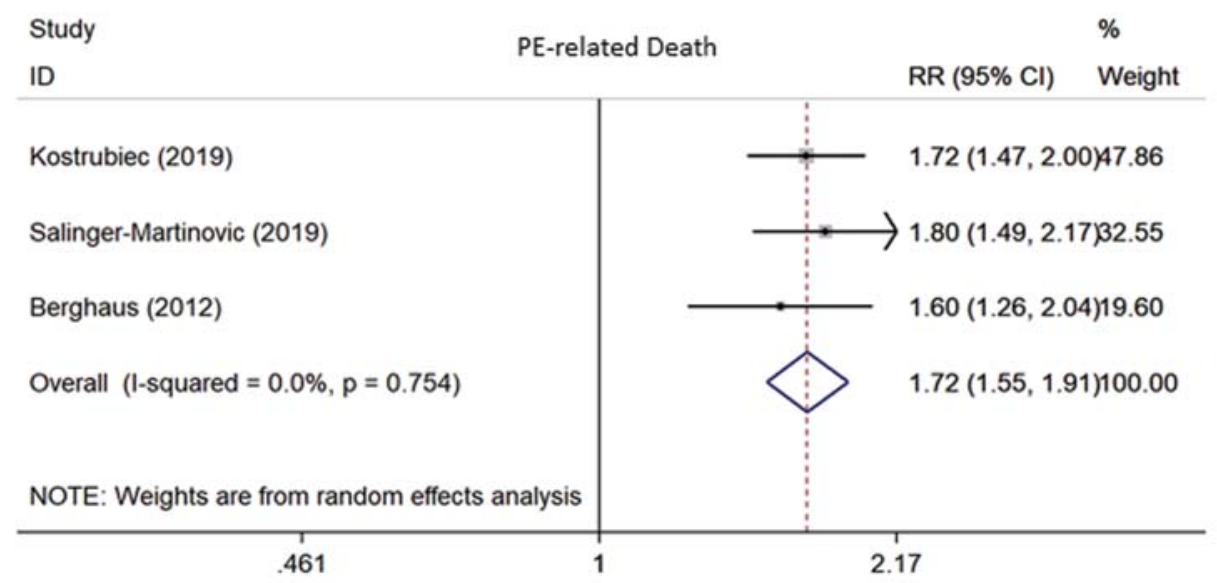

B

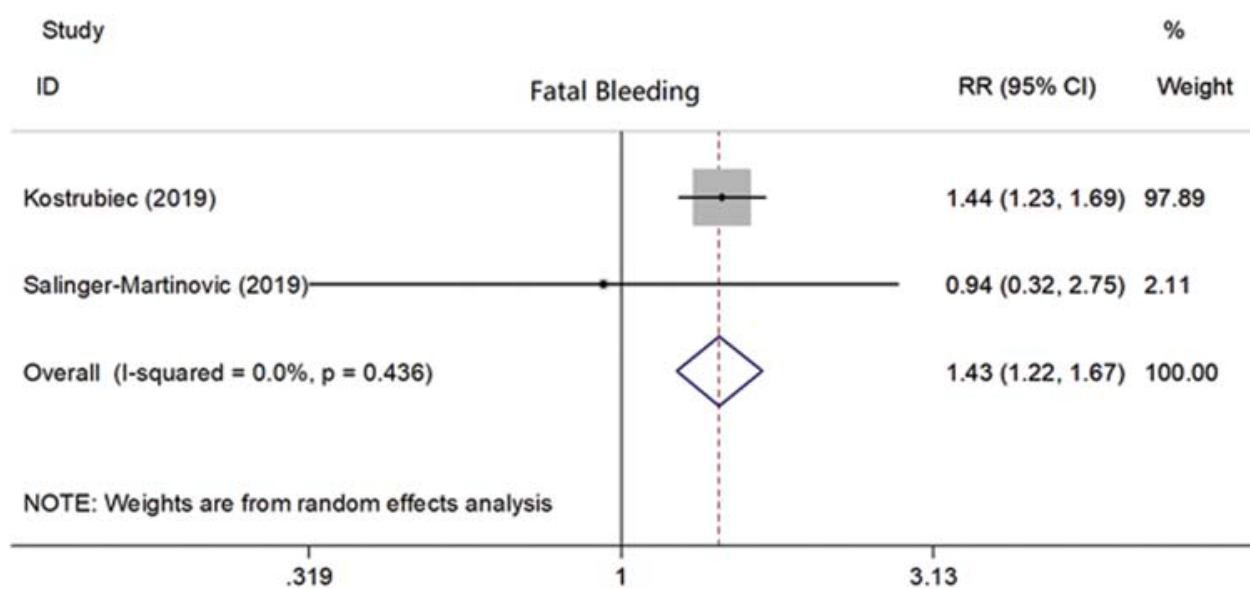

C

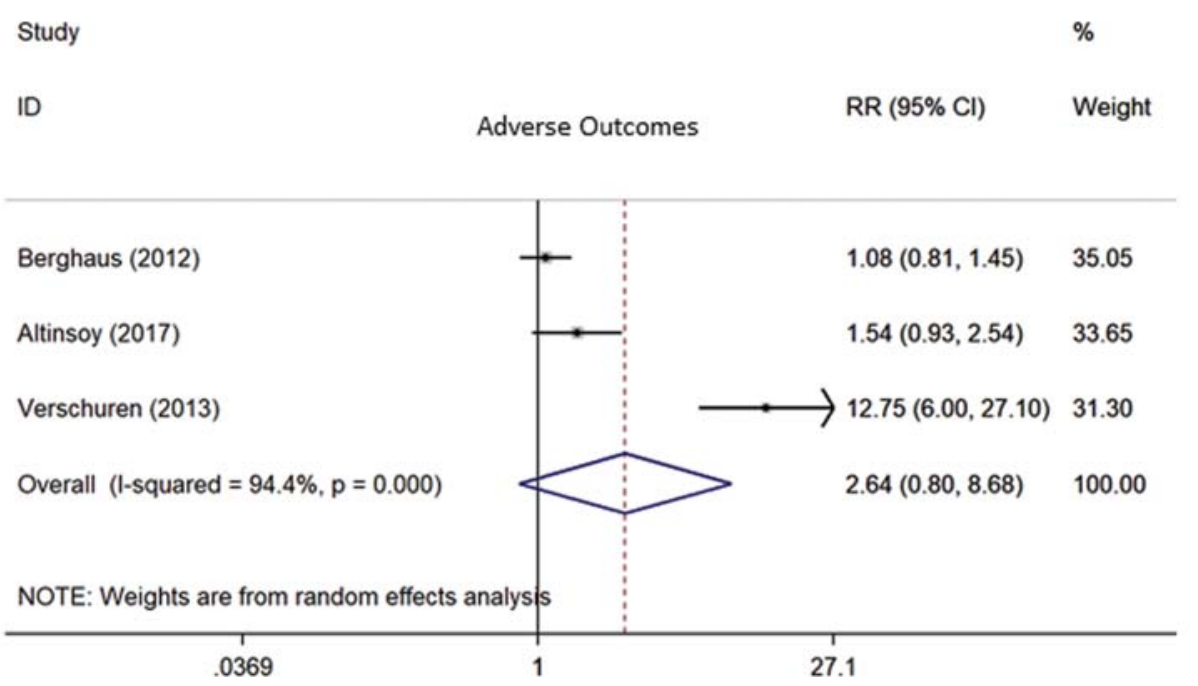

Fig. 5 Forest plots of pulmonary embolism (PE)-related death (A), fatal bleeding (B), and adverse outcomes (C) in pulmonary embolism (PE) patients with renal insufficiency. $95 \% \mathrm{Cl}, 95 \%$ confidence interval; $R R$, risk ratio.

multicenter registries, accurate renal function classification is not available for all the studies and studies without clear definitions of renal insufficiency were excluded from analysis. Second, stratifications of renal function were different in the selected studies and are impossible to transform, which may result in heterogeneity. Third, only normotensive patients were included in fatal bleeding analysis of severe renal insufficiency, which might be another source of heterogeneity. Finally, our study only included articles published in English language, which would increase the 
Table 3 Short-term clinical outcomes of normotensive PE patients

\begin{tabular}{|c|c|c|c|c|c|c|c|c|c|c|}
\hline \multirow[t]{3}{*}{ No. } & \multirow[t]{3}{*}{ Author } & \multirow{3}{*}{$\begin{array}{l}\text { Risk } \\
\text { level }\end{array}$} & \multirow{3}{*}{$\begin{array}{l}\text { RI } \\
\text { classification }\end{array}$} & \multirow{3}{*}{$\begin{array}{l}\text { RI number } \\
\text { (\%) }\end{array}$} & \multicolumn{6}{|c|}{ Outcome } \\
\hline & & & & & \multicolumn{2}{|c|}{ All-cause death } & \multicolumn{2}{|c|}{ Major bleeding } & \multicolumn{2}{|c|}{$\begin{array}{l}\text { Adverse out- } \\
\text { comes }\end{array}$} \\
\hline & & & & & $\begin{array}{l}\text { With } \\
\text { RI (\%) }\end{array}$ & $\begin{array}{l}\text { Without } \\
\text { RI (\%) }\end{array}$ & $\begin{array}{l}\text { With } \\
\text { RI (\%) }\end{array}$ & $\begin{array}{l}\text { Without } \\
\text { RI (\%) }\end{array}$ & $\begin{array}{l}\text { With } \\
\text { RI (\%) }\end{array}$ & $\begin{array}{l}\text { Without } \\
\text { RI (\%) }\end{array}$ \\
\hline 1 & $\begin{array}{l}\text { Kostrubiec } \\
\text { et al }\end{array}$ & $\begin{array}{l}\text { Low/ } \\
\text { intermediate }\end{array}$ & $\begin{array}{l}\mathrm{eGFR} \leq 60 \mathrm{~mL} / \\
\min \cdot 1.73 \mathrm{~m}^{-2}\end{array}$ & $\begin{array}{l}924 \\
(34.7 \%)\end{array}$ & $\begin{array}{l}95 \\
(10.3 \%)\end{array}$ & $\begin{array}{l}89 \\
(5.1 \%)\end{array}$ & - & - & - & - \\
\hline 2 & $\begin{array}{l}\text { Kostrubiec } \\
\text { et al }\end{array}$ & $\begin{array}{l}\text { Low/ } \\
\text { intermediate }\end{array}$ & $\begin{array}{l}\mathrm{eGFR} \leq 30 \mathrm{~mL} / \\
\min \cdot 1.73 \mathrm{~m}^{-2}\end{array}$ & $\begin{array}{l}116 \\
(4.4 \%)\end{array}$ & $\begin{array}{l}16 \\
(13.8 \%)\end{array}$ & $\begin{array}{l}168 \\
(6.6 \%)\end{array}$ & - & - & - & - \\
\hline 3 & $\begin{array}{l}\text { Altinsoy } \\
\text { et al }\end{array}$ & Normotensive & $\begin{array}{l}\mathrm{eGFR}<60 \mathrm{~mL} / \\
\mathrm{min} \cdot 1.73 \mathrm{~m}^{-2}\end{array}$ & $\begin{array}{l}38 \\
(38.4 \%)\end{array}$ & - & - & $\begin{array}{l}9 \\
(23.7 \%)\end{array}$ & $\begin{array}{l}7 \\
(11.5 \%)\end{array}$ & - & - \\
\hline 4 & $\begin{array}{l}\text { Kresoja } \\
\text { et al }\end{array}$ & $\begin{array}{l}\text { All but without } \\
\text { thrombolysis }\end{array}$ & $\begin{array}{l}\mathrm{eGFR}<60 \mathrm{~mL} / \\
\mathrm{min} \cdot 1.73 \mathrm{~m}^{-2}\end{array}$ & $\begin{array}{l}160 \\
(26.6 \%)\end{array}$ & - & - & - & - & $\begin{array}{l}13 \\
(8.1 \%)\end{array}$ & $\begin{array}{l}9 \\
(2.0 \%)\end{array}$ \\
\hline
\end{tabular}

Abbreviations: eGFR, estimated glomerular filtration rate; NA, not available; PE, pulmonary embolism; RI, renal insufficiency.

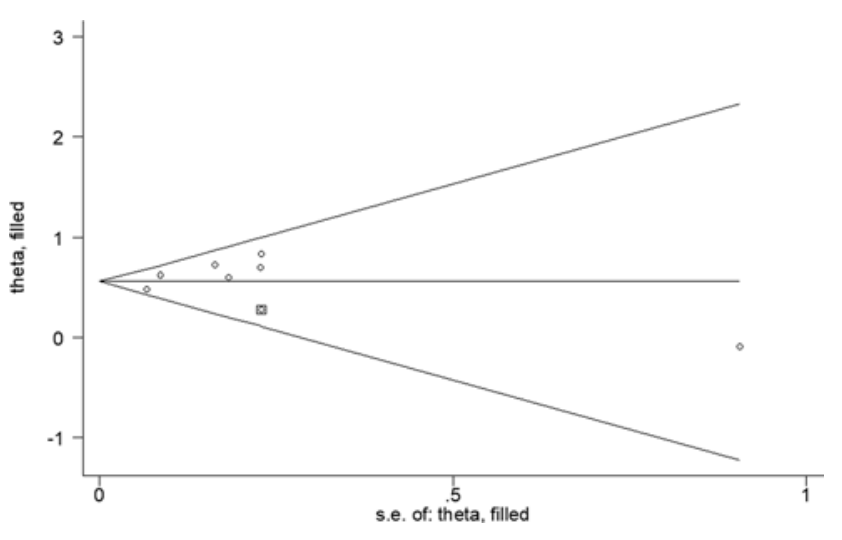

Fig. 6 Filled funnel plot with pseudo-95\% confidence limits.

publication bias. Subgroup analysis of normotensive PE patients could not be conducted due to the heterogeneity of studies. Further consideration of these patients should be focused as their characteristics and outcomes were different from high-risk PE.

\section{Conclusion}

Renal insufficiency is a predictor of short-term all-cause mortality in patients with acute PE. AKI and severe renal insufficiency had high risk for adverse short-term outcome in these patients. Our results my raise the attention in risk stratification and management of these patients in clinical practice.

\section{Funding}

This study is funded by The National Key Research and Development Program of China (No. 2016YFC0905600; 2016YFC0901104); CAMS Innovation Fund for Medical Sciences (CIFMS) (No. 2018-I2M-1-003); National Natural Science Foundation of China (No. 81570049; 81970058); Beijing Natural Science Foundation (No. 7152062).

Conflict of Interest

None declared.

\section{References}

1 Konstantinides SV, Meyer G, Becattini C, et al. ESC Scientific Document Group. 2019 ESC Guidelines for the diagnosis and management of acute pulmonary embolism developed in collaboration with the European Respiratory Society (ERS). Eur Heart J 2020;41(04):543-603

2 Aujesky D, Obrosky DS, Stone RA, et al. Derivation and validation of a prognostic model for pulmonary embolism. Am J Respir Crit Care Med 2005;172(08):1041-1046

3 Becattini C, Agnelli G, Lankeit M, et al. Acute pulmonary embolism: mortality prediction by the 2014 European Society of Cardiology risk stratification model. Eur Respir J 2016;48(03): 780-786

4 Vanni S, Nazerian P, Pepe G, et al. Comparison of two prognostic models for acute pulmonary embolism: clinical vs. right ventricular dysfunction-guided approach. J Thromb Haemost 2011;9 (10):1916-1923

5 Jiménez D, Aujesky D, Moores L, et al; RIETE Investigators. Simplification of the pulmonary embolism severity index for prognostication in patients with acute symptomatic pulmonary embolism. Arch Intern Med 2010;170(15):1383-1389

6 Barco S, Mahmoudpour SH, Planquette B, Sanchez O, Konstantinides SV, Meyer G. Prognostic value of right ventricular dysfunction or elevated cardiac biomarkers in patients with low-risk pulmonary embolism: a systematic review and meta-analysis. Eur Heart J 2019;40(11):902-910

7 Penaloza A, Roy PM, Kline J. Risk stratification and treatment strategy of pulmonary embolism. Curr Opin Crit Care 2012;18 (04):318-325

8 Goldhaber SZ, Visani L, De Rosa M. Acute pulmonary embolism: clinical outcomes in the International Cooperative Pulmonary Embolism Registry (ICOPER). Lancet 1999;353(9162):1386-1389

9 Monreal M, Falgá C, Valle R, et al; RIETE Investigators. Venous thromboembolism in patients with renal insufficiency: findings from the RIETE Registry. Am J Med 2006;119(12):1073-1079

10 Moher D, Liberati A, Tetzlaff J, Altman DG; PRISMA Group. Preferred reporting items for systematic reviews and meta-analyses: the PRISMA statement. PLoS Med 2009;6(07):e1000097

11 Kellum JA, Lameire N, Aspelin P, et al. KDIGO clinical practice guideline for acute kidney injury. Official J Int Society Nephrology 2012;2(01):124-138

12 Wells GA, Shea B, O'Connell D, et al. The Newcastle-Ottawa Scale (NOS) for assessing the quality of nonrandomised studies in metaanalyses. Available at: http://www.ohri.ca/programs/clinical_ epidemiology/oxford.asp. Accessed May 24, 2020

13 Schulman S, Angerås U, Bergqvist D, Eriksson B, Lassen MR, Fisher W; Subcommittee on Control of Anticoagulation of the Scientific 
and Standardization Committee of the International Society on Thrombosis and Haemostasis. Definition of major bleeding in clinical investigations of antihemostatic medicinal products in surgical patients. J Thromb Haemost 2010;8(01):202-204

14 Kostrubiec M, Pływaczewska M, Jiménez D, et al. The prognostic value of renal function in acute pulmonary embolism-a multicentre cohort study. Thromb Haemost 2019;119(01):140-148

15 Trimaille A, Marchandot B, Girardey M, et al. Assessment of renal dysfunction improves the simplified Pulmonary Embolism Severity Index (SPESI) for risk stratification in patients with acute pulmonary embolism. J Clin Med 2019;8(02):E160

16 Almeida S, Neiva JP, Bico P, et al. Renal function at admission as a prognostic marker in patients admitted for pulmonary embolism. Eur Heart J Acute Cardiovasc Care 2015;4:67

17 Salinger-Martinovic S, Dimitrijevic Z, Stanojevic D, et al. Are we calculated enough? Glomerular filtration rate as a predictor of intra-hospital prognosis in patients with pulmonary embolism. Eur Heart J 2019;40:4063

18 Kostrubiec M, Łabyk A, Pedowska-Włoszek J, et al. Assessment of renal dysfunction improves troponin-based short-term prognosis in patients with acute symptomatic pulmonary embolism. J Thromb Haemost 2010;8(04):651-658

19 Berghaus TM, Behr W, von Scheidt W, Schwaiblmair M. The Nterminal pro-brain-type natriuretic peptide based short-term prognosis in patients with acute pulmonary embolism according to renal function. J Thromb Thrombolysis 2012;33 (01):58-63

20 Altınsoy B, Öz İ̈, Örnek T, et al. Prognostic value of renal dysfunction indicators in normotensive patients with acute pulmonary embolism. Clin Appl Thromb Hemost 2017;23(06): 554-561

21 Kresoja KP, Ebner M, Rogge NIJ, et al. Prediction and prognostic importance of in-hospital major bleeding in a real-world cohort of patients with pulmonary embolism. Int J Cardiol 2019; 290:144-149

22 Jo JY, Lee MY, Lee JW, Rho BH, Choi WI. Leukocytes and systemic inflammatory response syndrome as prognostic factors in pulmonary embolism patients. BMC Pulm Med 2013;13:74

23 Keller K, Beule J, Balzer JO, Dippold W. Renal function as a cofactor for risk stratification and short-term outcome in acute pulmonary embolism. Exp Gerontol 2017;100:11-16

24 Agrawal N, Ramegowda RT, Patra S, et al. Predictors of inhospital prognosis in acute pulmonary embolism: keeping it simple and effective!. Blood Coagul Fibrinolysis 2014;25(05):492-500

25 Verschuren F, Bonnet M, Benoit M-O, et al. The prognostic value of pro-B-Type natriuretic peptide in acute pulmonary embolism. Thromb Res 2013;131(06):e235-e239

26 Murgier M, Bertoletti L, Darmon M, et al; RIETE Investigators. Frequency and prognostic impact of acute kidney injury in patients with acute pulmonary embolism. Data from the RIETE registry. Int J Cardiol 2019;291:121-126
27 Chang $\mathrm{CH}$, Fu CM, Fan PC, et al. Acute kidney injury in patients with pulmonary embolism: a population-based cohort study. Medicine (Baltimore) 2017;96(09):e5822

28 Wang IK, Shen TC, Muo CH, Yen TH, Sung FC. Risk of pulmonary embolism in patients with end-stage renal disease receiving long-term dialysis. Nephrol Dial Transplant 2017;32(08): 1386-1393

29 Kumar G, Sakhuja A, Taneja A, et al; Milwaukee Initiative in Critical Care Outcomes Research (MICCOR) Group of Investigators. Pulmonary embolism in patients with CKD and ESRD. Clin J Am Soc Nephrol 2012;7(10):1584-1590

30 Janus N, Mahé I, Launay-Vacher V, Laroche JP, Deray G. Renal function and venous thromboembolic diseases. J Mal Vasc 2016; 41(06):389-395

31 Berghaus TM, Schwaiblmair M, von Scheidt W. Renal biomarkers and prognosis in acute pulmonary embolism. Heart 2012;98(16): 1185-1186

32 Grams ME, Estrella MM, Coresh J, Brower RG, Liu KD; National Heart, Lung, and Blood Institute Acute Respiratory Distress Syndrome Network. Fluid balance, diuretic use, and mortality in acute kidney injury. Clin J Am Soc Nephrol 2011;6(05): 966-973

33 Siniscalchi C, Quintavalla R, Rocci A, et al; RIETE Investigators. Statin and all-cause mortality in patients receiving anticoagulant therapy for venous thromboembolism. Data from the RIETE registry. Eur J Intern Med 2019;68:30-35

34 Goto S, Turpie AGG, Zaghdoun A, et al. The influence of renal impairment on clinical outcomes in venous thromboembolism patients enrolled in GARFIELD- VTE. Research and Practice in Thrombosis and Haemostasis 2019;3:205-206

35 Lin MC, Streja E, Soohoo M, et al. Warfarin use and increased mortality in end-stage renal disease. Am J Nephrol 2017;46(04): 249-256

36 Ageno W, Casella IB, Han CK, et al. Baseline profile of patients treated for acute venous thromboembolism in routine clinical practice according to age and renal function in the RE-COVERY DVT_PE global cohort study. ESC Congress 2019 together with World Congress of Cardiology; 2019:2435

37 Levey AS, Coresh J, Balk E, et al; National Kidney Foundation. National Kidney Foundation practice guidelines for chronic kidney disease: evaluation, classification, and stratification. Ann Intern Med 2003;139(02):137-147

38 Levey AS, Stevens LA. Estimating GFR using the CKD Epidemiology Collaboration (CKD-EPI) creatinine equation: more accurate GFR estimates, lower CKD prevalence estimates, and better risk predictions. Am J Kidney Dis 2010;55(04):622-627

39 Fabbian F, Gallerani M, Pala M, et al. In-hospital mortality for pulmonary embolism: relationship with chronic kidney disease and end-stage renal disease. The hospital admission and discharge database of the Emilia Romagna region of Italy. Intern Emerg Med 2013;8(08):735-740 